\title{
Decline in excision circles requires homeostatic renewal or homeostatic death of naive $\mathrm{T}$ cells
}

\author{
Bas E. Dutilh, Rob J. de Boer* \\ Theoretical Biology and Bioinformatics Group, Utrecht University, Padualaan 8, 3584 CH Utrecht, The Netherlands
}

Received 5 March 2002; received in revised form 27 November 2002; accepted 4 December 2002

\begin{abstract}
When the TCR is formed in the thymus, fragments of DNA are excised from the T cell progenitor chromosome. These TCR rearrangement excision circles (TRECs) are stable, are not replicated in cell division and are therefore most frequent in naive T cells that have recently left the thymus. During life, the average TREC content of peripheral naive T cells decreases between one and two orders of magnitude in humans. It is generally believed that the age-dependent decrease in the production of naive $\mathrm{T}$ cells by the thymus is sufficient to explain the decrease in the TREC content. Here, we demonstrate that this decrease in thymic production is required, but it is not sufficient to explain the TREC data. Only if the decrease in thymic output is compensated by homeostasis can one explain the decrease in the TREC content. The homeostatic response can take two forms: when the total number of naive T cells declines, there could be an increase in the renewal rate or an increase of the average cellular lifespan.
\end{abstract}

(C) 2003 Elsevier Ltd. All rights reserved.

Keywords: Homeostasis; Excision circles; Naive T cells; Thymic involution; Ageing

\section{Introduction}

TCR rearrangement excision circles (TRECs) are circular DNA fragments, which are excised in the process of lymphocyte chromosome rearrangement during the development from progenitor to mature $\mathrm{T}$ cell in the thymus (Douek et al., 1998). TRECs are not replicated in cell division, but are divided randomly over the two daughter cells. The average TREC content of $T$ cells can be measured by specific PCR, and was shown to decrease almost 100-fold during a normal human lifetime in different kinds of T cells (Douek et al., 1998, 2001; Jamieson et al., 1999; Zhang et al., 1999). Since these studies measure TRECs in the total $\mathrm{T}$ cell population, and memory cells have much lower TREC numbers (Douek et al., 2001; Lewin et al., 2002), these observations can be confounded by a shift in the fractions of naive and memory cells over a person's lifetime (Fagnoni et al., 2000). The most conclusive data therefore is the study by Poulin et al. (1999), who find that the average TREC content per

\footnotetext{
*Corresponding author. Tel.: + 31-30-253-7560.

E-mail addresses: bedutilh@yahoo.com (B.E. Dutilh), r.j.deboer@bio.uu.nl (R.J. de Boer).
}

$\mathrm{CD} 4{ }^{+} \mathrm{CD} 45 \mathrm{RA}^{+} \mathrm{CD}_{62} \mathrm{~L}^{+}$naive $\mathrm{T}$ cell decreases more than ten-fold from 20- to 80-year olds. A similar decrease was reported by Douek et al. (2001), who measured TREC levels in sorted $\mathrm{CD}^{+} \mathrm{T}$ cells and, assuming that memory cells have a negligible contribution to the total TREC numbers, computed TRECs per $\mathrm{CD} 4{ }^{+} \mathrm{CD} 45 \mathrm{RO}^{-} \mathrm{CD} 27^{\text {high }}$ naive $\mathrm{T}$ cell.

Originally, assessment of the TREC content of the naive population was developed as an indicator of the production of recent thymic emigrants (RTEs, Douek et al., 1998). Because RTEs have the highest TREC levels, the decline in the TREC content with age is thought to reflect the age-related thymic involution (Steinmann et al., 1985). We will here confirm that a decrease in thymic production is required to explain the decrease in the average TREC content of naive $T$ cells during life, but we will also show that it is not sufficient. A decrease in thymic output decreases the influx of RTE with high TREC content, but this alone does not influence the average TREC content of the naive population. The only way the TREC content of a naive $\mathrm{T}$ cell can decrease is by division of the cell or intracellular degradation of the TREC. Here, we show that the decrease in the average TREC content of the naive population is determined by the decreasing thymic 
output, homeostatically countered by renewal and/or an increased lifespan of naive $T$ cells.

Whereas the function of the thymus decreases exponentially with age over two orders of magnitude (Steinmann et al., 1985), naive T cell numbers decrease much less with life. Fagnoni et al. (2000) have used a new marker for the naive $\mathrm{T}$ cell phenotype, CD95, and show that the numbers of circulating $\mathrm{CD} 4^{+}$and $\mathrm{CD} 8^{+}$ naive $\mathrm{T}$ cells decrease less than ten-fold from youngsters to centenarians. Because the thymic production decreases 100 -fold, this finding implies that naive $\mathrm{T}$ cell numbers are regulated homeostatically. However, it is unclear how this homeostasis is brought about. Theoretically, there are two possible mechanisms: a densitydependent renewal rate or a density-dependent death rate for the naive compartment (i.e. an increased proliferation rate or a decreased death rate when naive $\mathrm{T}$ cell numbers drop).

Division of naive $\mathrm{T}$ cells is a controversial issue. The classic view is that stimulation by the cognate antigen is the only way for naive $\mathrm{T}$ cells to proliferate, and that the process rapidly changes the phenotype of the cell from naive to memory. Recent evidence, however, suggests that naive $\mathrm{T}$ cells also readily divide when the cell numbers are severely depleted (e.g. after sublethal irradiation in mice; (Bender et al., 1999; Cho et al., 2000; Goldrath et al., 2000; Goldrath and Bevan, 1999; Kieper and Jameson, 1999; Marrack et al., 2000)). It remains unclear whether this process of depletioninduced proliferation actually contributes to homeostasis of the naive pool, since the expanding $\mathrm{T}$ cells typically obtain specific memory characteristics (Surh and Sprent, 2000; Cho et al., 2000).

The percentage of dividing naive $\mathrm{CD}^{+} \mathrm{T}$ cells (as defined by the expression of the $\mathrm{Ki}-67$ nuclear antigen) is increased when naive $\mathrm{T}$ cell numbers are low (Hazenberg et al., 2000b), e.g. in Dutch $\mathrm{HIV}^{+}$ patients and healthy HIV $^{-}$Ethiopians (Hazenberg et al., 2000a). These fractions are so high (e.g. 10\%) that the naive pool would be rapidly depleted if all $\mathrm{Ki}-67^{+}$naive $\mathrm{T}$ cells were to obtain a memory phenotype. Thus, it remains an open question whether truly naive $T$ cells divide, and whether this is regulated by homeostatic renewal.

The only other option for homeostasis is densitydependent regulation of the death rate. Freitas and Rocha (2000) stress that the maintenance of naive T cells in the periphery requires continuous TCR engagement by MHC molecules. Although Clarke and Rudensky (2000) argue that engagement of MHC molecules does not play an important role in the short term, they do admit that it is important in situations where naive cells survive for prolonged periods. Thus, competition by naive cells for survival signals from MHC molecules could act as a simple mechanism bringing about densitydependent cellular lifespans.
In this paper, we study a series of mathematical models that describe the kinetics of naive $\mathrm{T}$ cells and average TREC content on the basis of these possible presumptions. We compute the TREC frequencies in naive $\mathrm{T}$ cells throughout life for different scenarios for cell proliferation and cell death. We extend our previous work (Hazenberg et al., 2000a) by treating density-dependent proliferation and densitydependent death separately, and show that either of these mechanisms can explain the decline of the TREC content with age. In the total absence of density dependence, i.e. homeostasis, a decline in thymic production with age is not affecting the TREC content of naive $\mathrm{T}$ cells.

\section{Analyses}

\subsection{Model}

We develop a mathematical model of two ordinary differential equations describing the dynamics of naive T cells $N$ (either $\mathrm{CD} 4^{+}$or $\mathrm{CD} 8^{+} \mathrm{T}$ cells) and the total number of TRECs $T$ in the naive $\mathrm{T}$ cell population (Hazenberg et al., 2000a). Production of RTEs in the thymus is represented by parameter $\sigma(t)$, and $\rho(N)$ is the renewal term. Setting $\rho(N)=0$, we can study naive $\mathrm{T}$ cells without renewal. The cells are removed from the naive compartment at rate $\delta(N)$, which represents cell death plus priming by antigen (the latter changes the cells to the memory phenotype). For the total number of naive $\mathrm{T}$ cells, we therefore write

$\frac{\mathrm{d} N}{\mathrm{~d} t}=\sigma(t)+\rho(N) N-\delta(N) N$.

In the equation that describes the dynamics of the total number of TRECs $T$ in the naive T cell population, $c$ is the TREC level of an RTE. TRECs disappear from the population by intracellular degradation at rate $\delta_{I}$, or, together with their host cell, by death or antigenic priming at rate $\delta(N)$. In cell division, the TRECs are not replicated, and are randomly divided over the two daughter cells, so the total number of TRECs in the naive population is not affected by the renewal parameter $\rho(N)$ :

$\frac{\mathrm{d} T}{\mathrm{~d} t}=c \sigma(t)-\left(\delta(N)+\delta_{I}\right) T$.

Since the experimental data suggest the average number of TRECs per naive $T$ cell decreases with age, we rewrite Eq. (2) in terms of the average $A=T / N$ (Hazenberg et al., 2000a), i.e.

$\frac{\mathrm{d} A}{\mathrm{~d} t}=\frac{\sigma(t)}{N}(c-A)-\left(\delta_{I}+\rho(N)\right) A$.

If the dynamics of the $\mathrm{T}$ cells and of the average TREC levels are much faster than the slow thymic involution of 
$\sigma(t), A$ will approach a quasi-steady state (QSS)

$\bar{A}=\frac{c}{1+(N / \sigma(t))\left(\delta_{I}+\rho(N)\right)}$.

This QSS expression confirms the argumentation that in the absence of intracellular degradation and naive $\mathrm{T}$ cell renewal (i.e. $\delta_{I}+\rho(N)=0$ in Eq. (4)), there is no decline in the average TREC content, i.e. $\bar{A}=c$ (Hazenberg et al., 2000a).

\subsection{Parameters}

We will consider two different models in this paper. The "Renewal Model" contains density-dependent renewal of naive $\mathrm{T}$ cells, and a fixed death and priming rate. The "Death Model" contains density-dependent death, and either no renewal or a fixed renewal rate.

The thymic output $\sigma(t)$ is an exponentially decreasing function of age $t$ with a constant exponential rate $v$ (Steinmann et al., 1985):

$\sigma(t)=\sigma_{0} \mathrm{e}^{-v t}$.

The density-dependent renewal rate $\rho(N)$ in the Renewal Model can be modelled by a sigmoid Hill function with steepness parameter $k$. The value of $\rho(N)$ is halfmaximum when $N=h$, and has a maximum $\rho_{0}$, which is approached when cell numbers are low. By setting $k=0$, the renewal rate can be made non-homeostatic:

$\rho(N)=\frac{\rho_{0}}{1+(N / h)^{k}}$.

In the Death Model, the rate of death increases with increasing naive $\mathrm{T}$ cell numbers. A possible way to model this is

$\delta(N)=\delta_{0}(\varepsilon N)^{m}$.

The death rate can be made independent of the cell density by setting $m=0$. By Eq. (7), $\delta(N)$ approaches zero if $N \rightarrow 0$ (if $m>0$ ), allowing the cells to live forever; however, in our simulations the naive cell numbers are never in the very low range.

The parameters are adjusted such that for all proliferation and renewal terms, the models give the same steady state for a healthy 30 -year-old individual. On the basis of blood $\mathrm{T}$ cell numbers, blood volume and the percentage of $\mathrm{T}$ cells residing in the blood, Clark et al. (1999) calculate that a human adult has of the order of $10^{11}$ naive $\mathrm{CD}^{+}$and $\mathrm{CD} 8^{+} \mathrm{T}$ lymphocytes. To get $\delta(N)=\delta_{0}$ at $\bar{N}=10^{11}$ cells, we scale the homeostatic death function by setting $\varepsilon=10^{-11} /$ cell.

The TREC content of thymocytes does not change with age (Jamieson et al., 1999). Throughout life, naive $\mathrm{T}$ cells therefore leave the thymus with the same average TREC content. Because thymocytes divide after rearranging the TCR, most novel $\mathrm{T}$ cells emigrating from the thymus contain no TRECs. For example, Ye and Kirschner (2002) estimate an average TREC content of
0.118 TREC/cell from published data (Douek et al. 1998). The $c$ parameter of our model also represents the average TREC content of a naive $T$ cell emigrating from the thymus (i.e. RTE in the strictest sense of the word). Below we will show that the $c$ parameter can removed by appropriate scaling. Thus, representing our results in terms of $c$ (see Fig. 2(b) and Fig. 4(b) and (e)), we do not need a numerical estimate for $c$.

At low naive $\mathrm{T}$ cell counts, a maximum of $10 \%$ of the naive $\mathrm{T}$ cells was found to express $\mathrm{Ki}-67$ (Hazenberg et al., 2000a). It is difficult to link this percentage directly to the renewal rate, because estimates of the duration of the cell division process range from $6 \mathrm{~h}$ to 3 days. Taking one division per day as a gross estimate, we set $\rho_{0}=0.1 /$ day as our maximum proliferation rate (i.e. up to $10 \%$ of our T cells completes the division cycle in a day). Because the exponent of the involuting thymus $v \approx 0.05 /$ year throughout life (Steinmann et al., 1985), we need to set $\sigma_{0}=4.48 \times 10^{8}$ cells $/$ day to obtain the estimated thymic output of $\sigma(30$ years $)=10^{8}$ cells $/$ day in a healthy 30-year-old adult (Clark et al., 1999).

We incorporate the average turnover of a naive $\mathrm{T}$ cell, which is about 1000 days (Clark et al, 1999), by setting the normal death and priming rate $\delta\left(\left.N\right|_{30}\right.$ years $)=$ $0.001 /$ day in the Death Model, and the normal renewal rate $\rho\left(\left.N\right|_{30 \text { years }}\right)=0.001 /$ day in the Renewal Model. We obtain $\rho\left(\left.N\right|_{30 \text { years }}\right)=0.001 /$ day for different values of the steepness parameter $k$ by tuning $h$ $\left(h=1.01 \times 10^{9}\right.$ cells for $k=1$ to $h=3.17 \times 10^{10}$ cells for $k=4)$. This value of $\rho(N)$ requires that the death and priming rate is fixed at $\delta(N)=0.002 /$ day in the Renewal Model.

\subsection{Homeostasis is necessary}

We will start by investigating a model without homeostasis. Eq. (4) shows that we need at least renewal or intracellular degradation of TRECs to allow the average TREC levels to drop, so in this model we allow for a non-homeostatic renewal at rate $\rho$, and intracellular degradation $\delta_{I}$. The cell death and antigenic priming occurs at rate $\delta$. The naive lymphocyte population will approach

$\bar{N}=\frac{\sigma(t)}{\delta-\rho}$.

Substitution of $\bar{N}$ into the general QSS solution of the average TRECs (Eq. (4)) yields

$\bar{A}=\frac{c}{1+\left(\delta_{I}+\rho\right) /(\delta-\rho)}$.

The average TREC content of the naive T cells therefore goes to a fixed level, which is independent of the thymic output $\sigma(t)$, and is only determined by the (fixed) renewal and death rates (Hazenberg et al., 2000a). This result is independent of the values of the parameters $\rho, \delta$ 
or $\delta_{I}$. Thus, the model shows that the continued decline in the average number of TRECs per cell with age (Poulin et al., 1999; Douek et al., 2001) cannot be observed in a system without homeostasis, even if $\delta_{I}+$ $\rho>0$. As a consequence, renewal and/or death rates of naive $\mathrm{T}$ cells have to be density-dependent. This is the main point of this paper. Finally, note that the $c$ parameter merely scales the average TREC content. We next extend our previous work (Hazenberg et al., 2000a) by studying the two different possible mechanisms of homeostasis, renewal and death, separately.

\subsection{Homeostatic renewal}

First, we will assume that homeostasis is brought about by the adjustment of the renewal rate of naive $\mathrm{T}$ cells. As we have shown previously (Hazenberg et al., 2000a), homeostatic renewal can easily account for a realistic decrease in the average TREC content of the naive $\mathrm{T}$ cells. We generalize this Renewal Model here, studying different values of the homeostasis parameter $k$ (see Eq. (6)), while keeping the death and/or antigenic priming rate non-homeostatic $(m=0)$.

We study the model numerically, because the homeostatic $\rho(N)$ term is too complicated for analytical solution. By changing the steepness parameter $k$ in the renewal function (Eq. (6)), we can increase the strength of homeostasis from zero $(k=0)$ to very strong $(k$ large). Fig. 1 shows the influence of $k$ on the renewal rate functions. Only at very low $N$ does the number of dividing cells approach $10 \%$ (i.e. $\rho(N)=0.1 /$ day), which was the maximum level of naive $\mathrm{T}$ cells expressing Ki-67 (Hazenberg, et al., 2000a). For a normal healthy adult, with $N=10^{11}$ cells, the values for $\rho(N)$ are much lower, realistically close to the levels found in vivo (Hazenberg et al., 2000b).

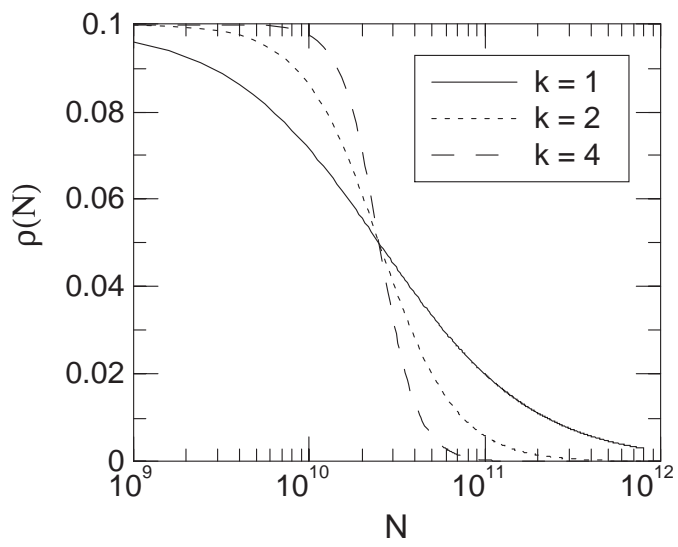

Fig. 1. Density-dependence of the renewal rate $\rho(N)$ for different values of $k$ ranging from 1 to 4 . For illustrative purposes, $h=$ $2.5 \times 10^{10}$ cells here, which differs slightly from the values of this parameter in the simulations in Fig. 2. $\rho_{0}=0.1 /$ day. Note that the horizontal axis has a logarithmic scale.
In Fig. 2, the two top panels show the naive $\mathrm{T}$ cells and their average TREC content vs. age for several values of the steepness parameter $k$. To make the models with different values of $k$ equivalent, we adjust $h$ (i.e. the naive count at which renewal is half-maximum), so that $\bar{N}=10^{11}$ at 30 years of age. Eq. (4) already proved that a decrease in the average TREC content requires that there is renewal or intracellular degradation $(\rho(N)+$ $\left.\delta_{I}>0\right)$. Since in this model $\rho(N)>0$, we can neglect intracellular degradation $\left(\delta_{I}=0 /\right.$ day $)$. This assumption has only a quantitative influence on the results: the major effect of $\delta_{I}$ is to shift the $A$-curve in Fig. 2(b) downwards (not shown). Because in all cases the cell numbers remain high relative to $h$, the differences
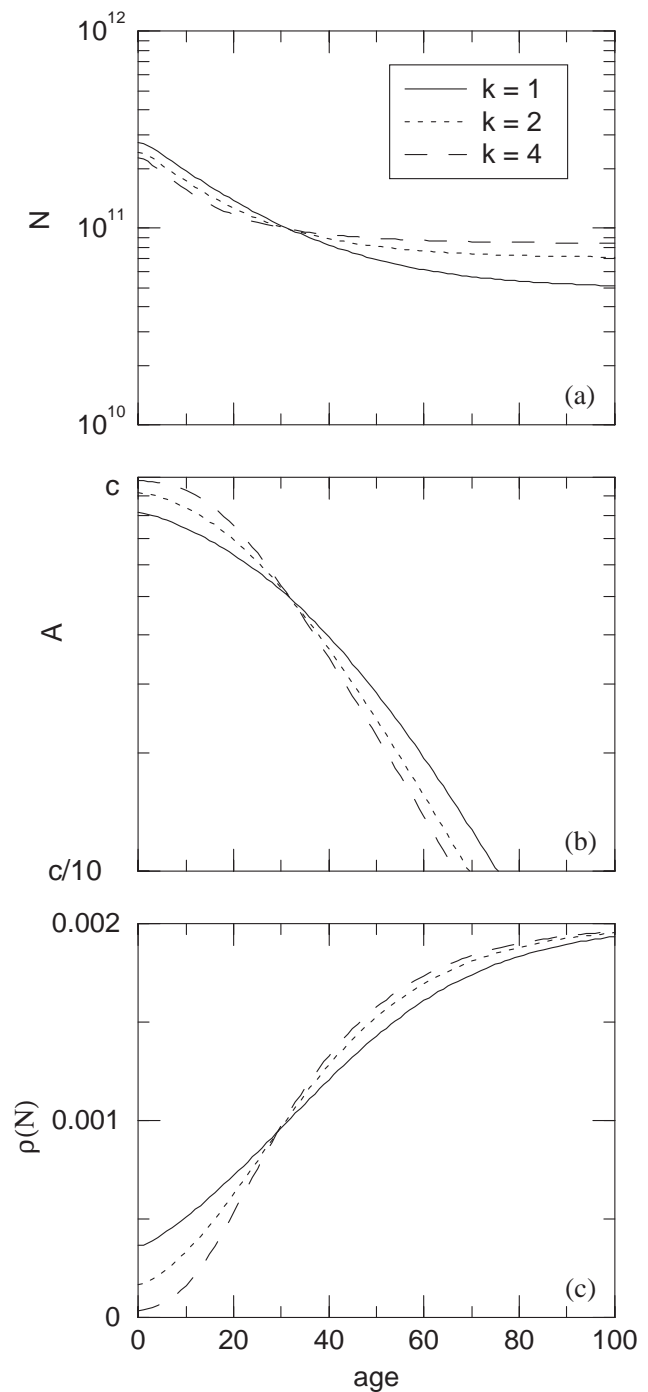

Fig. 2. The totals $N$ (a), average TREC content $A$ (b) and renewal rate $\rho(N)$ (c) of naive T cells in ageing individuals with increasing strength of the homeostatic renewal rate: $k$ ranges from 1 (weak homeostasis) to 4 (strong homeostasis). Parameters: $\sigma_{0}=4.48 \times 10^{8}$ cells $/$ day, $v=$ $0.05 /$ year, $\rho_{0}=0.1 /$ day, $\delta_{I}=0 /$ day, $\delta(N)=0.002 /$ day. To obtain a naive count of $10^{11}$ cells in a 30 -year old, the value of $h$ was chosen such that the renewal rate in a 30 -year old $\rho\left(\left.N\right|_{30 \text { years }}\right)=0.001 /$ day: $h_{1}=1.01 \times 10^{9}$ cells, $h_{2}=1.01 \times 10^{10}$ cells, $h_{4}=3.17 \times 10^{10}$ cells. 
in $\rho(N)$ are only minor (see Fig. 2(c)). Hence, the differences in the dynamics of the models with different values for $k$ remain small (Fig. 2(a) and (b)).

From these results, we can conclude that steady naive cell levels can be reached throughout life for any steepness of our homeostatic renewal function. The TREC frequencies $A$ show a realistic decrease of almost two orders of magnitude in all scenarios. To achieve steady naive cell levels, the renewal rate $\rho(N)$ increases during life (Fig. 2(c)), i.e. the division rates increase with age, and TRECs are diluted. To prevent excess growth of the naive cell numbers, the cell death and antigenic priming parameter $\delta$ has to be sufficiently large. Since the thymic output $\sigma(t) \approx 10^{8}$ cells/day in the healthy 30-year-old adult, a renewal rate of $\rho\left(\left.N\right|_{30 \text { years }}\right)=$ $0.001 /$ day (Clark et al., 1999) with a naive cell level $N=10^{11}$ cells requires that $\delta(N)=0.002 /$ day.

Due to the homeostatic renewal, $\rho(N)$ increases with age (see Fig. 2(c)). Douek et al. (2001) show that Ki-67 levels of naive $\mathrm{T}$ cells change very little with age, decreasing slightly in $\mathrm{CD}^{+}$naive $\mathrm{T}$ cells and increasing slightly in $\mathrm{CD} 8^{+}$naive $\mathrm{T}$ cells. Thus, there are no data to support the notion that naive division rates increase with age. This can be taken as an argument in favour of homeostatic death.

\subsection{Homeostatic death}

To consider the opposite extreme, let us now assume that the only source of naive lymphocytes is the thymic input $\sigma(t)$, i.e. we no longer allow for renewal $(\rho(N)=$ $0 /$ day). The possible Death Models can differ in the death and priming rate function $\delta(N)$ (Eq. (7)) by having different values of the steepness parameter $m$. The influence of $m$ on the death and priming rate function is illustrated in Fig. 3. Higher values of $m$ cause a stronger homeostasis, i.e. a death rate that increases more steeply with the population size.

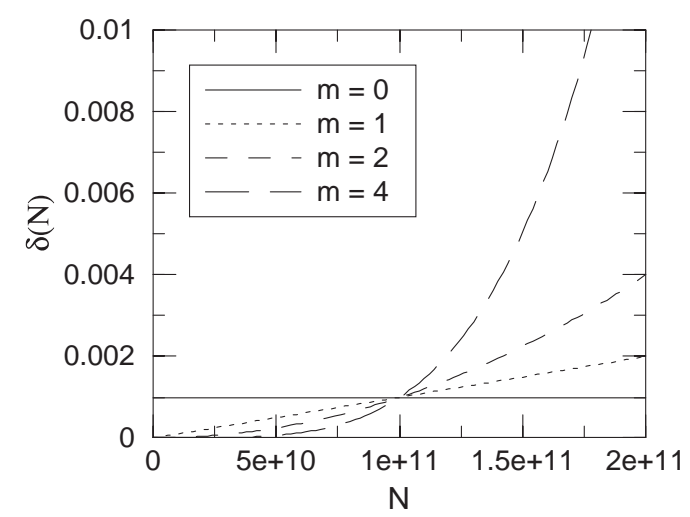

Fig. 3. Density dependence of the death and antigenic priming rate $\delta(N)$ for different values of $k$ ranging from 0 to 4 . The horizontal line shows density-independent death, as discussed in Section 2.3. $\delta_{0}=$ $0.001 /$ day and $\varepsilon=10^{-11} /$ cell.
Above, we showed that the simplest case, where the death and priming rate is fixed at $\delta=\delta_{0} \quad(m=0$, horizontal line in Fig. 3; no density dependence), cannot account for the TREC data because homeostasis is necessary (see Section 2.3). Thus, we start by studying models with a homeostatically adjusted death and priming rate (i.e. $m>0$ ). Combining the $\delta(N)$ function (Eq. (7)) with the naive T cell dynamics (Eq. (1)), results in the following QSS equations for the naive T cells:

$\bar{N}=(1 / \varepsilon) \sqrt[m+1]{\sigma(t) \varepsilon / \delta_{0}}$,

and for the average TREC content

$\bar{A}=\frac{c \sigma(t)^{m / m+1}}{\sigma(t)^{m / m+1}+\left(\delta_{I} / \varepsilon\right) \sqrt[m+1]{\varepsilon / \delta_{0}}}$.

Thus, the QSS value of the average TREC content per naive $\mathrm{T}$ cell $\bar{A}$ is a Michaelis-Menten function of the thymic output (more specifically, of $\sigma(t)^{m / m+1}$ ). Its behaviour is completely comprehensible in terms of the two parameters $c$ and $\left(\left(\delta_{I} / \varepsilon\right) \sqrt[m+1]{\varepsilon / \delta_{0}}\right)$. The higher the value of $m$, the more the exponent $m /(m+1)$ will approach one and the more the average TRECs will approach a simple Michaelis-Menten function of $\sigma(t)$. Since $\sigma(t)$ decreases with age, we also expect the average TREC content $A$ to decrease with age (see Fig. 4(b)). Panels (a) and (b) show the naive counts $N$ and the average TRECs per naive T cell $A$ in several models with density-dependent death and priming rates $(m=0$, i.e. no homeostasis, to $m=4$, i.e. strong homeostasis).

Provided the homeostatic death is strong (i.e. $m>2$ ), the total naive numbers $N$ decrease less than five-fold (Fig. 4(a)), which is in accordance with the experimental data (Fagnoni et al., 2000). The average TREC count in these naive $T$ cells decreases by more than one order of magnitude (Fig. 4(b)), which is also close to the value published in the literature (Poulin et al., 1999; Douek et al., 2001).

In the complete absence of naive T cell renewal $\rho(N)$ in the Death Model, intracellular degradation of TRECs $\delta_{I}$ is required to account for the decline in the average TREC levels with age. This can seen from Eq. (4), and can also be explained intuitively. As the naive $T$ cell numbers decrease, the expected lifespan of the lymphocytes increases (see Fig. 4(c)). When TRECs are degraded at a fixed rate $\delta_{I}$, the average TREC content of the naive $T$ cells decreases as the naive $T$ cell population gets older. The fact that TRECs persist for decades after thymectomy (Douek et al., 1998) suggests that the intracellular degradation is very slow. In order to obtain a realistic ten-fold decline of the TREC content of naive $\mathrm{T}$ cells with age, we have used a fairly high estimate of $\delta_{I}=0.001 /$ day in our simulations. For the current decline of thymic production in our model, lower $\delta_{I}$ values give a too slow and too late decline of the average TREC content. Summarizing, for sufficiently high intracellular TREC degradation, we can 

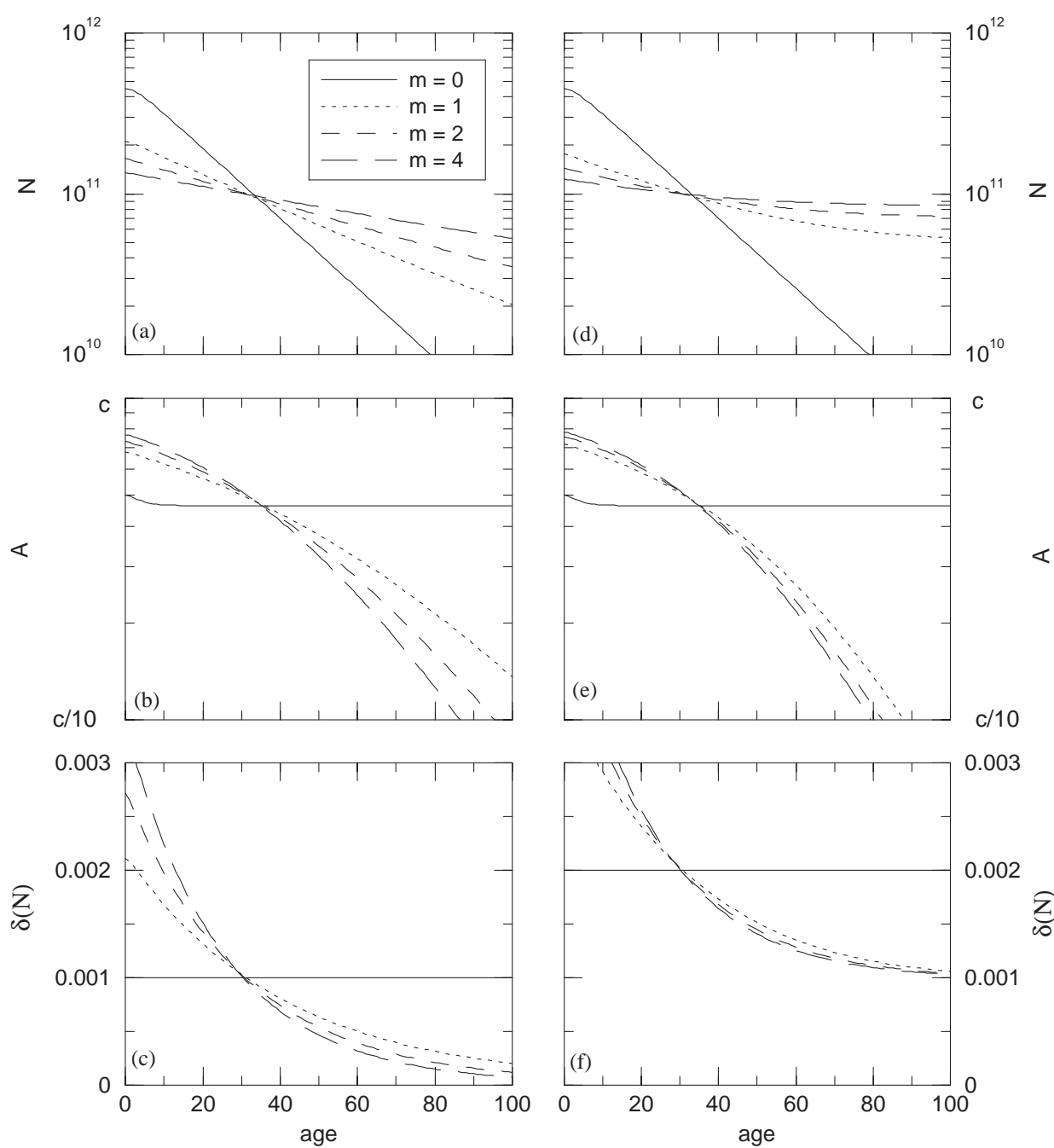

Fig. 4. The totals $N((\mathrm{a})$ and (d)), average TREC content $A$ ((b) and (e)) and the death and antigenic priming rate $\delta(N)((\mathrm{c})$ and (f)) of naive T cells in ageing individuals with increasing strength of the homeostatic death rate: $m$ ranges from 0 (no homeostasis) to 4 (strong homeostasis). Parameters: $\sigma_{0}=4.48 \times 10^{8}$ cells $/$ day, $v=0.05 /$ year, $\rho(N)=0 /$ day $((\mathrm{a})-(\mathrm{c}))$ or $\rho(N)=0.001 /$ day $((\mathrm{d})-(\mathrm{f})), \delta_{I}=0.001 /$ day $((\mathrm{a})-(\mathrm{c}))$ or $\delta_{I}=0 /$ day $((\mathrm{d})-(\mathrm{f}))$, $\delta_{0}=0.001 /$ day ((a)-(c)) or $\delta_{0}=0.002 /$ day ((d)-(f)), and $\varepsilon=10^{-11} /$ cell. With the values of $\delta_{0}$ used here, the naive count of a 30 -year old is always $10^{11}$ cells, so no further scaling is necessary.

explain both the homeostasis in the naive compartment and the observed decrease in the average TREC content of the naive cells with a model where naive T cells do not divide, and where homeostasis is maintained solely by the density-dependent death rate.

If the expected intracellular lifespan of TRECs is much longer than a 1000 days, one can still explain the data in models where homeostasis is solely due to density-dependent death, by allowing for a small density-independent proliferation rate. Assuming that naive $\mathrm{T}$ cells divide about once every 1000 days, i.e. fixing $\rho(N)=0.001 /$ day (by setting $\rho_{0}=0.002 /$ day and $k=0$ ), and setting $\delta_{I}=0$, is sufficient for a realistic loss of the TREC content of naive $\mathrm{T}$ cells with age in the death model (Fig. 4(d) and (e)). Intuitively, this is easy to understand because the small renewal rate results in more dilution of the TRECs if naive T cells live longer.

\section{Discussion}

Homeostasis of the naive $\mathrm{T}$ cell pool in healthy individuals is brought about by density-dependent regulation of the renewal rate and/or of the death rate of naive $T$ cells. Observations supporting the first hypothesis are the high renewal rates in organisms with depleted naive cell numbers (Cho et al., 2000; Goldrath et al., 2000). Because the number of TRECs per cell is diluted by renewal, the decrease in the average TREC levels in naive T cells with age also seems to support the naive $\mathrm{T}$ cell renewal hypothesis (Hazenberg et al., 2000a). Our Renewal Model does indeed show that it is possible to explain both the naive $\mathrm{T}$ cell dynamics and the average TREC levels by a homeostatically adjusted renewal rate (see Fig. 2). However, a number of open questions remain. 
Firstly, consider the high expansion rate of transferred naive $T$ cells found in mice with low peripheral $\mathrm{T}$ cell densities. There is a consensus that the dividing naive $T$ cells change to memory phenotype (see Surh and Sprent (2000) for review). Therefore, this form of naive $\mathrm{T}$ cell renewal seems to contribute to the memory $\mathrm{T}$ cell pool rather than to the naive $\mathrm{T}$ cell pool, and it seems inappropriate to call this "homeostatic renewal" of the naive $\mathrm{T}$ cell pool.

Secondly, Hazenberg et al. (2000b) argue that the high levels of $\mathrm{Ki}-67^{+}$naive $\mathrm{T}$ cells found in HIV-infected individuals, where naive counts are low, are not an indication of homeostasis. Antiretroviral treatment of the patients brings the level of Ki-67 expression down, while the naive $\mathrm{T}$ cell levels fail to normalize. This is not what one would expect if homeostasis were the only process involved. The authors suggest that a state of general activation of the immune system rather than homeostasis is the cause of the high division rate in untreated $\mathrm{HIV}^{+}$patients. This could also be the case with the healthy Ethiopians with increased Ki-67 levels studied by Hazenberg et al. (2000a).

The results of the transfer experiments and the Ki-67 data described above do not support the hypothesis of homeostasis-controlled naive $\mathrm{T}$ cell renewal. However, we still do not know how to explain them. Why does the general state of hyperactivation brought about by HIV (Hazenberg et al., 2000b) also influence naive T cells? In what respect does this mechanism resemble the mechanism of depletion-triggered proliferation in irradiated mice? Our models only show that homeostasis of the naive $T$ cell population is necessary to explain its slow decline with age and the continued decline of its average TREC content, and do not make a statement on the mechanistic nature of the homeostasis.

As can be seen in Fig. 2(b), the average TREC levels in the Renewal Model decrease only slightly during the first years of life, starting to decline in late puberty. Zhang et al. (1999) also found a shoulder shape in the dynamics of the average TREC content vs. age per peripheral blood mononuclear cell. The point of decrease, which lies slightly earlier in their findings, is approached better if we use stronger homeostasis (higher values of $k$, see Fig. 2(b)). In the current investigations, the shoulder shape was due solely to the use of a saturated $(k=1)$ or sigmoid $(k>1)$ density dependence of the renewal function $\rho(N)$ (see Eq. (6)). Comparable shoulder-shaped dynamics were obtained in the Death Model when a sigmoid function was implemented instead of the power function for $\delta(N)$ (results not shown). It is not known how, or even whether, renewal and death rates depend on the total population size. We have therefore chosen for a conventional Hill function for the density dependent renewal, and for a simple power function for the density-dependent death. We do not expect the results to depend strongly on the choice of these functions.

Returning to the issue of hyperactivation in $\mathrm{HIV}^{+}$ patients (Hazenberg, et al., 2000a), there is an interesting corollary in the Death Model. Since homeostasis in the Death Model is brought about by allowing for long lifespans when the cell numbers are low, homeostasis is expected to fail when the priming rate is increased too much. Our death and priming rate $\delta(N)$ in fact consists of a priming component $\pi$ and a true density-dependent death component, e.g.

$\delta(N)=\pi+\delta_{0}(\varepsilon N)^{m}$.

If, during an infection, an individual experiences a small increase in the priming rate $\pi$, this would increase $\delta(N)$ and deplete the naive T cell pool somewhat. This can be homeostatically compensated for by decreasing the true death $\delta_{0}(\varepsilon N)^{m}$. However, if $\pi$ is increased too much, homeostasis will fail because perfect homeostatic compensation requires the true death term to decrease below zero. When homeostasis is at its maximum (i.e. $\left.\delta_{0}(\varepsilon N)^{m} \rightarrow 0\right)$, naive cells have infinitely long expected lifespans, but nevertheless become primed at rate $\pi$. The steady-state level of the naive $\mathrm{T}$ cells becomes inversely proportional to the priming rate (i.e. $N=\sigma(t) / \pi$ ). This could indeed account for the loss of naive $T$ cells, i.e. the apparent lack of homeostasis, in diseases like $\mathrm{HIV}^{+}$ infection (Hazenberg et al., 2000a), and rheumatoid arthritis (Koetz et al., 2000; Ponchel et al., 2002). Shortening the expected lifespan of naive $T$ cells by increasing the priming or death rate, should increase their TREC content however. Thus, to explain the loss of naive T cells and their lower TREC content in HIV-1 infected patients, one would additionally require increased division and/or decreased thymic production (Hazenberg et al., 2000a).

Two recent modelling papers addressed the TREC content of T cells in patients infected with HIV-1. Lewin et al. (2002) develop a model by writing equations for $\mathrm{TREC}^{+}$and TREC ${ }^{-} \mathrm{T}$ cells. They predict the effect of the various parameters of the model by computing partial derivatives. Although the model is very different from the ones developed here, and before (Hazenberg et al., 2000a), the results are very similar. Because their basic model has no density dependence they indeed find that changing thymic output has no effect on the average TREC content. Ye and Kirschner (2002) develop a much more complicated model with several homeostatic functions. It is therefore not surprising that their model can explain the loss of TRECs with age. However, Ye and Kirschner (2002) argue that our previous results were biased because we previously only showed results setting the intracellular loss of TRECs $\delta_{I}=0$. Because thymectomized patients continue to have TREC $^{+} \mathrm{T}$ cells decades after the thymectomy (Douek et al., 1998), we indeed think $\delta_{I}$ is small. 
Importantly, however, we have shown here that one can explain all data allowing for either a small intracellular decay and/or for infrequent naive $\mathrm{T}$ cell division, provided naive $\mathrm{T}$ cell numbers are homeostatically regulated.

\section{Acknowledgements}

We thank José Borghans, Mette Hazenberg and Frank Miedema for discussion and comments, and Sheila $\mathrm{McNab}$ for linguistic advice. BD was financially supported by the Dutch AIDS Foundation (Grant 4025).

\section{References}

Bender, J., Mitchell, T., Kappler, J., Marrack, P., 1999. CD4 ${ }^{+}$T cell division in irradiated mice requires peptides distinct from those responsible for thymic selection. J. Exp. Med. 190, 367-374.

Cho, B.K., Rao, V.P., Ge, Q., Eisen, H.N., Chen, J., 2000. Homeostasis-stimulated proliferation drives naive $\mathrm{T}$ cells to differentiate directly into memory T cells. J. Exp. Med. 192, 549-556.

Clark, D.R., De Boer, R.J., Wolthers, K.C., Miedema, F., 1999. T cell dynamics in HIV-1 infection. Adv. Immunol. 73, 301-327.

Clarke, S.R., Rudensky, A.Y., 2000. Survival and homeostatic proliferation of naive peripheral $\mathrm{CD} 4+\mathrm{T}$ cells in the absence of self peptide: MHC complexes. J. Immunol. 165, 2458-2464.

Douek, D.C., McFarland, R.D., Keiser, P.H., Gage, E.A., Massey, J.M., Haynes, B.F., Polis, M.A., Haase, A.T., Feinberg, M.B., Sullivan, J.L., Jamieson, B.D., Zack, J.A., Picker, L.J., Koup, R.A., 1998. Changes in thymic function with age and during the treatment of HIV infection. Nature 396, 690-695.

Douek, D.C., Betts, M.R., Hill, B.J., Little, S.J., Lempicki, R., Metcalf, J.A., Casazza, J., Yoder, C., Adelsberger, J.W., Stevens, R.A., Baseler, M.W., Keiser, P., Richman, D.D., Davey, R.T., Koup, R.A., 2001. Evidence for increased T cell turnover and decreased thymic output in HIV infection. J. Immunol. 167, 6663-6668.

Fagnoni, F.F., Vescovini, R., Passeri, G., Bologna, G., Pedrazzoni, M., Lavagetto, G., Casti, A., Franceschi, C., Passeri, M., Sansoni, P., 2000. Shortage of circulating naive $\mathrm{CD}^{+} \mathrm{T}$ cells provides new insights on immunodeficiency in aging. Blood 95, 2860-2868.

Freitas, A.A., Rocha, B., 2000. Population biology of lymphocytes: the flight for survival. Annu. Rev. Immunol. 18, 83-111.

Goldrath, A.W., Bevan, M.J., 1999. Low-affinity ligands for the TCR drive proliferation of mature CD8 $+\mathrm{T}$ cells in lymphopenic hosts. Immunity 11, 183-190.

Goldrath, A.W., Bogatzki, L.Y., Bevan, M.J., 2000. Naive T cells transiently acquire a memory-like phenotype during homeostasisdriven proliferation. J. Exp. Med. 192, 557-564.

Hazenberg, M.D., Otto, S.A., Stuart, J.W., Verschuren, M.C., Borleffs, J.C., Boucher, C.A., Coutinho, R.A., Lange, J.M.,
DeWit, T.F., Tsegaye, A., Van Dongen, J.J., Hamann, D., DeBoer, R.J., Miedema, F., 2000a. Increased cell division but not thymic dysfunction rapidly affects the T-cell receptor excision circle content of the naive $\mathrm{T}$ cell population in HIV-1 infection. Nat. Med. 6, 1036-1042.

Hazenberg, M.D., Stuart, J.W., Otto, S.A., Borleffs, J.C., Boucher, C.A., DeBoer, R.J., Miedema, F., Hamann, D., 2000b. T-cell division in human immunodeficiency virus (HIV)-1 infection is mainly due to immune activation: a longitudinal analysis in patients before and during highly active antiretroviral therapy (HAART). Blood 95, 249-255.

Jamieson, B.D., Douek, D.C., Killian, S., Hultin, L.E., ScriptureAdams, D.D., Giorgi, J.V., Marelli, D., Koup, R.A., Zack, J.A., 1999. Generation of functional thymocytes in the human adult. Immunity 10, 569-575.

Kieper, W.C., Jameson, S.C., 1999. Homeostatic expansion and phenotypic conversion of naive $\mathrm{T}$ cells in response to self peptide/MHC ligands. Proc. Natl Acad. Sci. USA 96, 13306-13311.

Koetz, K., Bryl, E., Spicksen, K., O'Fallon, W.M., Goronzy, J.J., Weynand, C.M., 2000. T cell homeostasis in patients with rheumatoid arthritis. Proc. Natl Acad. Sci. 97, 9203-9208.

Lewin, S.R., Ribeiro, R.M., Kaufmann, G.R., Smith, D., Zaunders, J., Law, M., Solomon, A., Cameron, P.U., Cooper, D., Perelson, A.S., 2002. Dynamics of T cells and TCR excision circles differ after treatment of acute and chronic HIV infection. J. Immunol. 169, 4657-4666.

Marrack, P., Bender, J., Hildeman, M., Jordan, D.A.N., Mitchell, T., Murakami, M., Sakamoto, A., Schaefer, B.C., Swanson, B., Kappler, J., 2000. Homeostasis of $\alpha \beta \mathrm{TCR}^{+} \mathrm{T}$ cells. Nat. Immunol. 1, 107-111.

Ponchel, F., Morgan, A.W., Bingham, S.J., Quinn, M., Buch, M., Verburg, R.J., Henwood, J., Douglas, S.H., Masurel, A., Conagham, P., Gesinde, M., Taylor, J., Markham, A.F., Emery, P., Van Laar, J.M., Isaacs, J.D., 2002. Dysregulated lymphocyte proliferation and differentiation in patients with rheumatoid arthritis. Blood 0, 0 .

Poulin, J.F., Viswanathan, M.N., Harris, J.M., Komanduri, K.V., Wieder, E., Ringuette, N., Jenkins, M., McCune, J.M., Sekaly, R.P., 1999. Direct evidence for thymic function in adult humans. J. Exp. Med. 190, 479-486.

Steinmann, G.G., Klaus, B., Muller-Hermelink, H.K., 1985. The involution of the ageing human thymic epithelium is independent of puberty. A morphometric study. Scand. J. Immunol. 22, 563-575

Surh, C.D., Sprent, J., 2000. Homeostatic T cell proliferation. How far can T cells be activated to self-ligands? J. Exp. Med. 192, F9-F14.

Ye, P., Kirschner, D.E., 2002. Reevaluation of T cell receptor excision circles as a measure of human recent thymic emigrants. J. Immunol. 169, 4968-4979.

Zhang, L., Lewin, S.R., Markowitz, M., Lin, H.H., Skulsky, E., Karanicolas, R., He, Y., Jin, X., Tuttleton, S., Vesanen, M., Spiegel, H., Kost, R., Van Lunzen, J., Stellbrink, H.J., Wolinsky, S., Borkowsky, W., Palumbo, P., Kostrikis, L.G., Ho, D.D., 1999. Measuring recent thymic emigrants in blood of normal and HIV-1infected individuals before and after effective therapy. J. Exp. Med. 190, 725-732. 